\title{
Shear and dielectric responses of propylene carbonate, tripropylene glycol, and a mixture of two secondary amides
}

Gainaru, Catalin ; Hecksher, Tina; Olsen, Niels Boye; Böhmer, Roland; Dyre, J. C.

Published in:

Journal of Chemical Physics

DOI:

$10.1063 / 1.4740236$

Publication date:

2012

Document Version

Publisher's PDF, also known as Version of record

Citation for published version (APA):

Gainaru, C., Hecksher, T., Olsen, N. B., Böhmer, R., \& Dyre, J. C. (2012). Shear and dielectric responses of propylene carbonate, tripropylene glycol, and a mixture of two secondary amides. Journal of Chemical Physics, 137(6), 064508-1 - 064508-10. https://doi.org/10.1063/1.4740236

\section{General rights}

Copyright and moral rights for the publications made accessible in the public portal are retained by the authors and/or other copyright owners and it is a condition of accessing publications that users recognise and abide by the legal requirements associated with these rights.

- Users may download and print one copy of any publication from the public portal for the purpose of private study or research.

- You may not further distribute the material or use it for any profit-making activity or commercial gain.

- You may freely distribute the URL identifying the publication in the public portal.

\section{Take down policy}

If you believe that this document breaches copyright please contact rucforsk@kb.dk providing details, and we will remove access to the work immediately and investigate your claim. 


\section{AIP $\begin{gathered}\text { molowanat } \\ \text { chemical Physics }\end{gathered}$}

Shear and dielectric responses of propylene carbonate, tripropylene glycol, and a mixture of two secondary amides

Catalin Gainaru, Tina Hecksher, Niels Boye Olsen, Roland Böhmer, and Jeppe C. Dyre

Citation: J. Chem. Phys. 137, 064508 (2012); doi: 10.1063/1.4740236

View online: http://dx.doi.org/10.1063/1.4740236

View Table of Contents: http://jcp.aip.org/resource/1/JCPSA6/v137/i6

Published by the American Institute of Physics.

Additional information on J. Chem. Phys.

Journal Homepage: http://jcp.aip.org/

Journal Information: http://jcp.aip.org/about/about_the_journal

Top downloads: http://jcp.aip.org/features/most_downloaded

Information for Authors: http://jcp.aip.org/authors

\section{ADVERTISEMENT}

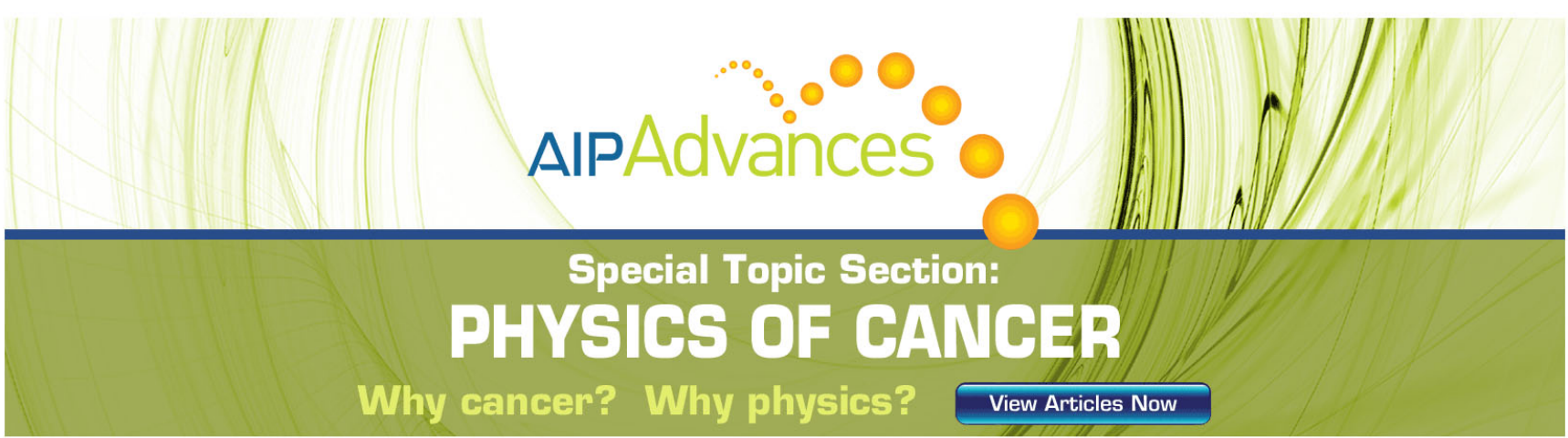




\title{
Shear and dielectric responses of propylene carbonate, tripropylene glycol, and a mixture of two secondary amides
}

\author{
Catalin Gainaru, ${ }^{1}$ Tina Hecksher, ${ }^{2}$ Niels Boye Olsen, ${ }^{2}$ Roland Böhmer, ${ }^{1}$ \\ and Jeppe C. Dyre ${ }^{2}$ \\ ${ }^{1}$ Fakultät für Physik, Technische Universität Dortmund, 44221 Dortmund, Germany \\ ${ }^{2}$ DNRF Centre "Glass and Time," IMFUFA, Department of Sciences, Roskilde University, Postbox 260, \\ DK-4000 Roskilde, Denmark
}

(Received 31 May 2012; accepted 17 July 2012; published online 14 August 2012)

\begin{abstract}
Propylene carbonate and a mixture of two secondary amides, $\mathrm{N}$-methylformamide and $\mathrm{N}$ ethylacetamide, are investigated by means of broadband dielectric and mechanical shear spectroscopy. The similarities between the rheological and the dielectric responses of these liquids and of the previously investigated tripropylene glycol are discussed within a simple approach that employs an electrical circuit for describing the frequency-dependent behavior of viscous materials. The circuit is equivalent to the Gemant-DiMarzio-Bishop model, but allows for a negative capacitive element. The circuit can be used to calculate the dielectric from the mechanical response and vice versa. Using a single parameter for a given system, good agreement between model calculations and experimental data is achieved for the entire relaxation spectra, including secondary relaxations and the Debye-like dielectric peak in the secondary amides. In addition, the predictions of the shoving model are confirmed for the investigated liquids. () 2012 American Institute of Physics. [http://dx.doi.org/10.1063/1.4740236]
\end{abstract}

\section{INTRODUCTION}

Dielectric spectroscopy is the most commonly employed method for the investigation of supercooled liquids due to the particularly large dynamic range that it permits to be accessed. ${ }^{1-5}$ In spite of its broad applicability this method is usually not able, just by itself, to provide detailed information regarding the microscopic mechanisms leading to the complex relaxation pattern specific for each glass former. Hence, conventional dielectric studies are often supplemented by investigations via other techniques which offer alternative perspectives on the relaxation phenomena. Using such a combined approach one can gain information on the underlying molecular dynamics not only by comparing the time scale of the structural fluctuations (the $\alpha$-process) ${ }^{6}$ but also by comparing the spectral shapes probed with different methods. ${ }^{7-9}$ Depolarized light scattering ${ }^{8,10-12}$ and nuclear magnetic resonance (NMR) (Refs. 8, 9, 11, and 13) are among the techniques which are often employed in that respect because, similar to dielectric spectroscopy, they provide access to molecular orientational correlation functions.

Mechanical spectroscopy, yet another experimental probe, is also able to yield data in a broad frequency range. ${ }^{14-16}$ This technique was used early on for studying the rubbery behavior of polymers. ${ }^{17,18}$ Later developments transformed it into a method widely used for the investigation of supercooled liquids and soft matter, in general. ${ }^{15,19}$ The first model connecting the frequency dependent dielectric permittivity with the frequency dependent viscoelastic constants was proposed by Gemant, who employed an equivalent electrical circuit to characterize the macroscopic behavior of liquids. ${ }^{20}$ Physical relations similar to the ones obtained by Gemant, but based on microscopic details, were derived by DiMarzio and Bishop in $1974 .{ }^{21}$ In this model, called in the following the Gemant-DiMarzio-Bishop (GDB) model, the friction term that appears in the rotational diffusion equation of the Debye theory ${ }^{22}$ is chosen to be frequency dependent, to account for the viscoelastic behavior of liquids containing rotating dipole moments. Suffering from some shortcomings, the GDB model was reformulated several times, ${ }^{15,23-25}$ with the intention to describe the behavior of viscous materials in a range extending up to frequencies at which the mechanical response becomes purely elastic. The high-frequency shear modulus $G_{\infty}$ characterizing this regime is a crucial parameter for several theoretical approaches ${ }^{26}$ including the shoving model, ${ }^{27}$ which aims at describing the temperature dependence of the structural relaxation in supercooled liquids. In 2005 Niss et al. concluded that the GDB model generally gives a qualitatively satisfactory description of the interrelation between dielectric and shear mechanical data, especially for systems with low dielectric strength. ${ }^{28}$

One of the systems that we studied in the course of the present work is the glass former propylene carbonate (PC). Due to its particularly large molecular dipole moment, this van der Waals liquid is well investigated with dielectric spectroscopy from temperatures close to its boiling point ${ }^{29}$ down to the cryogenic regime, ${ }^{30}$ but mechanical data are sparse. PC belongs to a special class of glass formers with no obvious secondary Johari-Goldstein $\beta$-peak ${ }^{31}$ in their dielectric loss spectra. For these systems a power-law contribution with a negative exponent, the so-called excess wing, ${ }^{32,33}$ emerges close to the glass transition temperature $T_{g}$ on the high-frequency flank of the $\alpha$-peak. Regardless of the debated microscopic origin of the excess wing, ${ }^{34,35}$ it was recently demonstrated that the spectral shape of this feature 
exhibits a high degree of similarity when probed with techniques such as dielectric spectroscopy, field-cycling NMR, or light scattering. ${ }^{7,8,36}$ On the other hand, to our knowledge, an excess wing was never reported to occur in mechanical studies of molecular liquids.

In addition, we will report on the mechanical response for a mixture of two secondary amides. These hydrogen-bonded liquids share a spectral shape similar to that of monoalcohols, for which a monodispersive (Debye-like) peak with anomalously high amplitude dominates the dielectric spectra. ${ }^{37}$ For monohydroxy alcohols it is well documented that this dielectric feature in not the structural relaxation and it has remained undetected in measurements with most other techniques ${ }^{38-41}$ including shear mechanical spectroscopy. ${ }^{42,43}$ In this work we test whether or not the strong dielectric loss peak observed for secondary amides ${ }^{37}$ displays any rheological signature.

In order to connect mechanical and dielectrical relaxations, in the present paper we start from postulating a simple electrical circuit equivalent to the GDB model. With numerous aspects of this model already discussed, ${ }^{15,23-25}$ this circuit is deliberately not given a specific physical interpretation. In this sense the circuit represents a "black box" approach with the main purpose to provide a minimal model of the coupling between mechanical and electrical relaxations in supercooled liquids. This approach allows us to re-parameterize the GDB model in a manner such that all except one of the model's input parameters are directly experimentally accessible quantities. The resulting model is tested for three liquids, two of them being investigated in this work dielectrically and as well as rheologically over wide frequency ranges. Consistent with previously briefly noted results ${ }^{28}$ we provide evidence that the model fits data for a wide range of glass formers excellently if a negative capacitance is allowed for. We argue that this sign of the capacitance is not necessarily unphysical. We also argue that the model accounts for the most striking features of dielectric relaxation in secondary amides. Finally, we show that the viscoelastic data taken in the case of the present work conform to the prediction which the shoving-model ${ }^{27}$ provides for the non-Arrhenius temperature dependence of the relaxation time.

The paper is organized as follows: in Sec. II some experimental details are given, and the circuit model is introduced in Sec. III. The experimental results and their analysis are presented in Sec. IV. The discussion of the time constants, the conversion of the spectra using the circuit model, and a test of the shoving model for PC and the amide mixture is carried out in Sec. V. The conclusions are given in Sec. VI.

\section{EXPERIMENTAL DETAILS}

Propylene carbonate (PC, stated chemical purity 99\%), $\mathrm{N}$-methylformamide (NMF, 99\%), and $\mathrm{N}$-ethylacetamide (NEA, 99\%) were purchased from Sigma Aldrich. The liquids were measured as received, without further treatment. Neat NMF and NEA show a high tendency of crystallization which is reduced if the two amides are mixed. ${ }^{37}$ The shear measurements were performed using the piezoelectric shear modulus gauge described in Ref. 44. Both the shear and the dielectric transducers were inserted into the same cryostat to attain identical temperature stabilization. The description of the overall experimental setup and its limitations are given in Refs. 24, 45, and 46. The dielectric data for PC and for the mixture that were recorded in the present work are in good agreement with those of earlier investigations..$^{29,32,37}$

\section{THEORETICAL BACKGROUND}

\section{A. The circuit approach for modeling of linear responses}

In an experiment some parameters are controlled - the input (the applied field) as well as parameters that are kept fixed - while others vary in response to the input. If $\langle A(t)\rangle$ is the measured output ( $A$ is assumed to have zero equilibrium value), the linear response $R(t)$ to an input field $\gamma(t)$ is defined via the following equation:

$$
\langle A(t)\rangle=\int_{0}^{\infty} R(\tau) \dot{\gamma}(t-\tau) d \tau .
$$

This convolution integral expresses linearity and causality, i.e., that the measured output depends only on the input before the measurement. The convention used here differs from what is often used (e.g., in Ref. 47) where the "response function" is defined as the time-derivative of $R(t)$, while $R(t)$ itself is termed the "relaxation function." Defining $R(t)$ as the response function has the advantage that $R(t)$ has the dimension of output over input, the same dimension as that of the frequency-dependent response function $R(\omega)$.

Measured response functions traditionally have different names, depending on which quantities are controlled (input) and which are measured (output). Thus, in mechanical measurements the terms modulus and relaxation spectrum are used if strain is controlled and stress is measured, whereas if stress is controlled and strain is measured one refers to the (creep) compliance and retardation spectrum. ${ }^{18}$ Mathematically, the two representations are equivalent. However, for a step input and subsequent measurement of the output as a function of time, the relation between the two is quite complicated (see, for instance, Ref. 18) because it requires knowledge of the entire spectrum. If the input is oscillatory and the output is measured as a function of frequency, on the other hand, the complex modulus and the compliance functions are simply the inverse of each other. These examples are taken from mechanical spectroscopy, but the terminology has been carried over to the electrical analogue, where charge plays the role of shear displacement, current plays the role of shear rate, and voltage plays the role of shear stress.

A convenient approach to the modeling of a linear system is to regard it as defined via one or more so-called "energy bonds." These model how the environmental variables interact with the system (see, e.g., Ref. 48 and references quoted therein). An energy bond is a pair of conjugate variables the product of which has the dimension of energy transferred per time. Examples are shear stress and shear rate, pressure and rate of volume change, temperature variation and entropy current, or electrical voltage and current. Because of the close formal analogy between different energy bonds, all systems can be modeled by electrical circuit elements. Crucially, the 
energy bond formalism allows for connecting different bonds through transformer elements that convert one type of energy into another. This feature, which is used below, shows the strength of the formalism.

Electrical equivalent circuit models do not necessarily give insight into the microscopic processes of the modeled system, although in practice the individual circuit elements often do have a microscopic interpretation. In any case, a circuit model can be always viewed as a phenomenological "macro-to-macro" model that relates different measured quantities. To be useful such models should be as simple as possible. Elements of electrical circuits such as resistors and capacitances model certain behavior of matter, depending on what plays the role of generalized voltage and of generalized current. If, for instance, the conjugate pair of energy bond variables is shear stress and strain rate, a capacitance models an elastic response and a resistor models a viscous flow response. The Maxwell model for viscoelastic behavior is an example of how these elements can be combined to mimic the response of a viscous liquid; in the electrical language the Maxwell model is given by a resistor and a capacitor in parallel - the force (voltage) is common to both elements.

There are several advantages to this type of modeling. First of all, linearity as well as basic physical principles such as conservation laws, causality, and positive dispersion are automatically obeyed in network models. A second advantage is the following: It is often stated that one must "compare modulus responses to modulus responses." There is, however, just one overall circuit (as there is only one dynamics at the microscopic level). From this one can extract either its complex, frequency-dependent modulus or its compliance: The circuit's response is calculated from that of its individual elements by the application of Kirchhoff's laws. The currents through and voltage drops across individual elements are uniquely determined from the current (or voltage) input applied at the circuit's outer terminals, the relation of which determines the circuit's overall response function. Each individual element has a unique relation between its current and voltage. For instance, in the circuit discussed in Sec. III B via Stokes' law the element representing the rotational movement of the dipoles has an impedance (voltage over current) that is proportional to the viscosity (shear stress over strain rate). In transforming the mechanical responses to an electrical response, the only thing to remember is that shear displacement corresponds to charge, strain rate to current, and shear force to voltage. In this way the discussion of which mechanical response function to compare to which electrical response function becomes obsolete.

\section{B. The conversion between rheological and dielectric data}

Following previous considerations, ${ }^{20,49}$ the approach introduced here models the material under investigation using an electrical circuit similar to the one sketched in Fig. 1. Such a combination of simple electrical elements is able to mimic the structural relaxation in liquids. The frequency-dependent

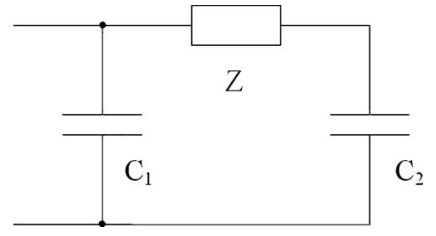

FIG. 1. The electrical circuit analogue used for the transformation between dielectric and shear data. Apart from the two capacitive elements $C_{1}$ and $C_{2}$, an impedance $Z \propto \eta$ is used.

complex permittivity is given by

$$
\varepsilon^{*}(\omega)=C_{1} / C_{0}+\frac{C_{2} / C_{0}}{1+i \omega Z C_{2}},
$$

with $C_{0}$ denoting the geometrical capacitance and $\omega$ denoting the angular frequency. As a simple example, note that equivalence with the Debye expression ${ }^{22}$ is obtained if the impedance $Z$ is chosen as a frequency independent resistor $R$. In this case the equivalent complex permittivity of the material reduces to the well-known Debye equation, ${ }^{22}$ provided that

(i) $C_{1} / C_{0}=\varepsilon_{\infty}$ models the high-frequency response and

(ii) $C_{2} / C_{0}=\Delta \varepsilon$ parameterizes the relaxation strength of the structural process.

With $Z=R$, the product $R C_{2} \equiv \tau$ represents the relaxation time which, according to the Debye theory, is proportional to the viscosity of the liquid. ${ }^{22}$

To connect dielectric and mechanical properties, in the following the impedance $Z^{*}(\omega)$ is chosen to be frequency dependent and proportional to the complex frequency dependent viscosity, $\eta^{*}(\omega)$, so that

$$
Z^{*}(\omega)=\lambda \eta^{*}(\omega) / C_{0} .
$$

Here, $\lambda$ is an electro-viscoelastic material constant expressed in units of $\mathrm{Pa}^{-1}$. Under these conditions, and using $i \omega \eta^{*}(\omega)=G^{*}(\omega)$, Eq. (3) can be written as

$$
\begin{aligned}
\varepsilon^{*}(\omega) & =C_{1} / C_{0}+\frac{C_{2} / C_{0}}{1+i \omega \lambda\left(C_{2} / C_{0}\right) \eta^{*}(\omega)} \\
& =A+\frac{B}{1+\lambda B G^{*}(\omega)}
\end{aligned}
$$

with $C_{1} / C_{0} \equiv A$ and $C_{2} / C_{0} \equiv B$.

Equation (4) connects the complex permittivity, $\varepsilon^{*}(\omega)$ $=\varepsilon^{\prime}(\omega)-i \varepsilon^{\prime \prime}(\omega)$, and the complex shear modulus, $G^{*}(\omega)$ $=G^{\prime}(\omega)+i G^{\prime \prime}(\omega)$. The two quantities are experimentally accessed via dielectric and mechanical spectroscopy, respectively. We note that the microscopic GDB model provides an expression identical to Eq. (4). ${ }^{21}$ In the original GDB approach the constant $\lambda B$ that appears in this equation is identified with $4 \pi R^{3} / k_{B} T$, with $R$ denoting the effective radius of the molecule and $T$ denoting the absolute temperature.

It is obvious that the simple GDB model, for which the parameters $A$ and $B$ turn out to be given by relations (i) and (ii), cannot lead to a good transformation between permittivity and shear modulus data, since the second term in Eq. (4) does not reduce to zero in the high-frequency limit where 
$\varepsilon^{*}(\omega \rightarrow \infty)=C_{1} / C_{0}$ should hold. This shortcoming is due to the fact that in this frequency range the real part of the shear modulus of the liquid is finite, i.e., $G^{\prime}(\omega \rightarrow \infty)=G_{\infty}$. Therefore, to alleviate this problem we require that in Eq. (4) the values of the two constants $A$ and $B$ need to be adjusted in a way such that the two following physical conditions for the real part of $\varepsilon^{*}$ are fulfilled:

(a) $\varepsilon^{\prime}(\omega \rightarrow 0)=\varepsilon_{\infty}+\Delta \varepsilon=A+B$,

(b) $\quad \varepsilon(\omega \rightarrow \infty)=\varepsilon_{\infty}=A+\frac{B}{1+\lambda B G_{\infty}}$.

The above equations were obtained from Eq. (4) exploiting that $G^{\prime}=0$ and $G^{\prime \prime}=0$ for $\omega \rightarrow 0$ and $G^{\prime}=G_{\infty}$ and $G^{\prime \prime}=0$ for $\omega \rightarrow \infty$. Relation (b) indicates the presence of a non-negligible elastic rotational contribution to the dielectric constant in the high-frequency limit, as previously discussed in Ref. 28. The values for $A$ and $B$ that comply with these two conditions are

$$
\begin{aligned}
& A(\lambda)=\varepsilon_{\infty}+\frac{1}{2}\left(\Delta \varepsilon-\sqrt{\Delta \varepsilon^{2}+\frac{4 \Delta \varepsilon}{\lambda G_{\infty}}}\right), \\
& B(\lambda)=\frac{1}{2}\left(\Delta \varepsilon+\sqrt{\Delta \varepsilon^{2}+\frac{4 \Delta \varepsilon}{\lambda G_{\infty}}}\right) .
\end{aligned}
$$

Inserting these expressions into Eq. (4), the two components of $\varepsilon^{*}(\omega)$ are seen to be related to $G^{\prime}(\omega)$ and $G^{\prime \prime}(\omega)$ via

$$
\begin{aligned}
& \varepsilon^{\prime}(\omega)=A(\lambda)+\frac{B(\lambda)\left[1+\lambda B(\lambda) G^{\prime}(\omega)\right]}{\left[1+\lambda B(\lambda) G^{\prime}(\omega)\right]^{2}+\left[\lambda B(\lambda) G^{\prime \prime}(\omega)\right]^{2}}, \\
& \varepsilon^{\prime \prime}(\omega)=\frac{\lambda B(\lambda)^{2} G^{\prime \prime}(\omega)}{\left[1+\lambda B(\lambda) G^{\prime}(\omega)\right]^{2}+\left[\lambda B(\lambda) G^{\prime \prime}(\omega)\right]^{2}} .
\end{aligned}
$$

Conversely, for the two components of the complex shear modulus one obtains

$$
\begin{aligned}
G^{\prime}(\omega) & =\frac{1}{\lambda}\left\{\frac{\varepsilon^{\prime}-A(\lambda)}{\left[\varepsilon^{\prime}-A(\lambda)\right]^{2}+\left(\varepsilon^{\prime \prime}\right)^{2}}-\frac{1}{B(\lambda)}\right\}, \\
G^{\prime \prime}(\omega) & =\frac{1}{\lambda} \frac{\varepsilon^{\prime \prime}}{\left[\varepsilon^{\prime}-A(\lambda)\right]^{2}+\left(\varepsilon^{\prime \prime}\right)^{2}} .
\end{aligned}
$$

In these transformation equations, Eqs. (6) and (7), only one parameter, $\lambda$, is adjustable while the values for $\varepsilon_{\infty}, \Delta \varepsilon$, and $G_{\infty}$ that enter into the relations for $A$ and $B$ can be taken directly from the experimental data.

For very small relaxation strengths, $\Delta \varepsilon \ll \lambda G_{\infty}$, parameter $B$ in Eq. (5) approaches $\Delta \varepsilon$ and likewise $A$ becomes equal to $\varepsilon_{\infty}$ independent of $G_{\infty}$. For triphenylethylene, tetramethyltetraphenyltrisiloxane, or squalane, $\Delta \varepsilon$ is quite small ( 0.05 at $249 \mathrm{~K}, 0.2$ at $211 \mathrm{~K}$, and 0.015 at $167 \mathrm{~K}$, respectively) and this may explain why the simple GDB approach works satisfactorily for these systems. ${ }^{24,28}$ For squalane the GDB model applies well for the entire relaxation spectrum including both the $\alpha$ - and the $\beta$-process, see Fig. 9.7 of Ref. 24 .

On the other hand, for systems with large dielectric strength, the parameter $A$ may turn negative if the parameter $\lambda$ is tuned to below a certain limit $\lambda_{\min }$. In this case, even though the (phenomenological) transformation between the two susceptibilities might still work well, a negative value for $A$, or for the capacitor $C_{1}$ in Fig. 1, will require a non-standard interpretation of the circuit model as briefly outlined in Sec. V B. From Eq. (5) one obtains

$$
\lambda_{\min }=\frac{\Delta \varepsilon}{G_{\infty} \varepsilon_{\infty}\left(\varepsilon_{\infty}+\Delta \varepsilon\right)}
$$

by setting $A=0$. Since $G_{\infty}$ and $\varepsilon_{\infty}$ do not vary much among different molecular liquids, $\lambda_{\min }$ should not be too different for systems like PC, tripropylene glycol (TPG), or the NEANMF mixture, for which $\Delta \varepsilon \gg \varepsilon_{\infty}$.

\section{SHEAR MECHANICAL EXPERIMENTS AND DATA ANALYSIS}

In Figs. 2(a) and 2(b) we present the real and the imaginary part, respectively, of the shear modulus for PC, as measured for temperatures between $173 \mathrm{~K}$ and $155 \mathrm{~K}$ in a frequency range covering six decades. We note that Schröter and Donth ${ }^{14}$ previously reported shear measurements for this material at a single temperature and in a more restricted frequency range. Their results are compatible with the present data when analyzing the time constants, but not the amplitude. In our experiments $G_{\infty}$ is $30 \%$ higher than the value reported in Ref. 14.

The inflection point in $G^{\prime}(v)$, which marks the crossover from the viscous response (at low frequencies) to the elastic response (at high frequencies) of PC, shifts through the accessible frequency window in a rather small temperature range of less than $20 \mathrm{~K}$, see Fig. 2(a). This is due to the high fragility of this material. ${ }^{50}$ A high-frequency plateau in $G^{\prime}(v)$, with amplitude $G_{\infty}$, is observable only at the lowest temperature $T=155 \mathrm{~K}$. For temperatures larger than $155 \mathrm{~K}, G_{\infty}$ can, in principle, be determined as the area under the $G^{\prime \prime}(v)$ curves in Fig. 2(b). If data in a very broad frequency range are available, this can be done directly using the Kramers-Kronig relation $G_{\infty}=\frac{2}{\pi} \int_{0}^{\infty} \frac{G^{\prime \prime}(\omega)}{\omega} d \omega$. In the present situation we found it more advisable to use the following procedure instead. The

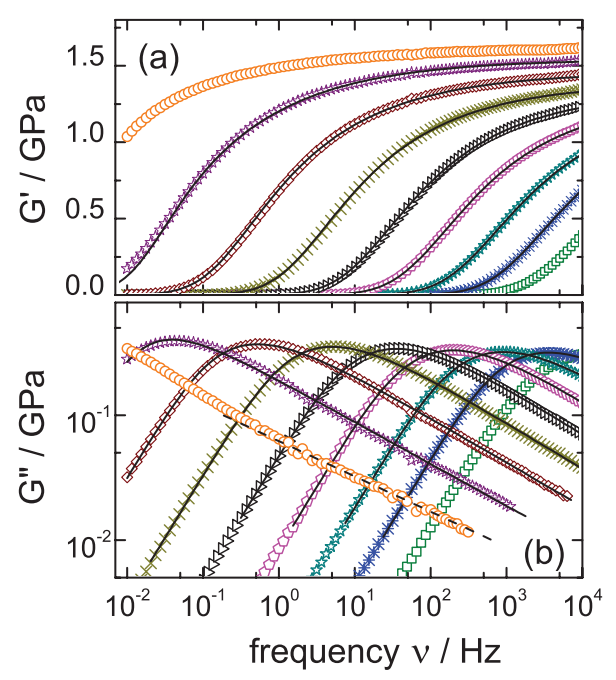

FIG. 2. (a) The real and (b) the imaginary part of the shear modulus as a function of frequency for PC measured every $2 \mathrm{~K}$ between $171 \mathrm{~K}$ and $155 \mathrm{~K}$. The solid lines are fits using Eqs. (9) and (10). The dashed line highlights the contribution of the excess wing. 
shear modulus data were fitted using the recently introduced step-response function ${ }^{51}$

$$
\phi(t)=\Gamma\left[\frac{\beta}{\alpha},\left(\frac{t}{\tau_{0}}\right)^{\alpha}\right] / \Gamma\left[\frac{\beta}{\alpha}\right],
$$

with $\Gamma(\xi)=\int_{0}^{\infty} x^{\xi-1} \exp (-x) d x$ denoting Euler's gamma function and $\Gamma(\xi, y)=\int_{y}^{\infty} x^{\xi-1} \exp (-x) d x$ denoting the upper incomplete gamma function. ${ }^{52}$ Here, $\beta$ parameterizes the high-frequency side of the peak maximum loss according to $G^{\prime \prime}(v) \propto v^{-\beta}$, while $\alpha$ is a parameter characterizing the overall peak width, see Ref. 51 for details. The given power-law behavior follows directly from performing the Laplace transform of $\phi(t)$, which gives the complex shear modulus:

$$
G^{*}(\omega)=i \omega G_{\infty} \int_{0}^{\infty} \phi(t) \exp (-i \omega t) d t
$$

As shown in Ref. 51, $\phi(t)$ reduces to a single exponential, corresponding to a Maxwell process for $\alpha=\beta=1$, to a ColeDavidson function for $\alpha=1$, or to a stretched exponential (Kohlrausch function) for $\alpha=\beta$. The reason for choosing the function given by Eq. (9) is its enhanced flexibility and, above all, its ability to reliably reproduce the frequency dependence on the low frequency side of the broad elastic modulus loss peak, where the loss is proportional to frequency $v$. We note that Eqs. (9) and (10) provide a better fit than the frequently used Havriliak-Negami function, ${ }^{51,53}$ and this was also found for the present data.

The quality of the numerical interpolation of the PC data via Eqs. (9) and (10) is close to perfect, as the solid lines in Fig. 2 indicate. This fitting procedure allowed us to extract $G_{\infty}$, to determine the mean shear relaxation time, $\tau_{\text {shear }}$ $\equiv \int_{0}^{\infty} \phi(t) d t,{ }^{54}$ and also to parameterize the spectral shape via the coefficients $\alpha$ and $\beta$.

For PC we find that $\alpha=0.75 \pm 0.05$ and $\beta=0.33 \pm 0.01$ when temperature is varied from $157 \mathrm{~K}$ to $167 \mathrm{~K}$. The temperature independence of the two parameters indicates that time-temperature superposition applies well for the shear data of PC. This is also illustrated in Fig. 4(a) where all the loss spectra scale to a single curve after normalization of the peak amplitude and of the characteristic frequency. As observed in Fig. 4(b) and discussed more at the end of this section, such a scaling is not possible for the mixture of the two secondary amides.

The results for $\tau_{\text {shear }}$ of PC are plotted in Fig. 5 as a function of the inverse temperature. At the lowest temperature, $T=155 \mathrm{~K}$, the presence of an additional feature on the high-frequency side of the peak maximum becomes visible if the data are plotted on a logarithmic scale, see Fig. 2(b). The crossover between the high-frequency flank of the main peak and a power-law with a lower exponent (highlighted by the dashed line) indicates that an excess wing is present in the shear data of PC, as also observed in its dielectric spectra. ${ }^{32}$

Shear mechanical data were also acquired for a mixture of the secondary amides NMF and NEA. Since we were not able to supercool any of the two neat substances, we decided to perform measurements on the mixture of these two compounds that was previously investigated by Wang and Richert using dielectric spectroscopy. ${ }^{37}$ As indicated by these au-

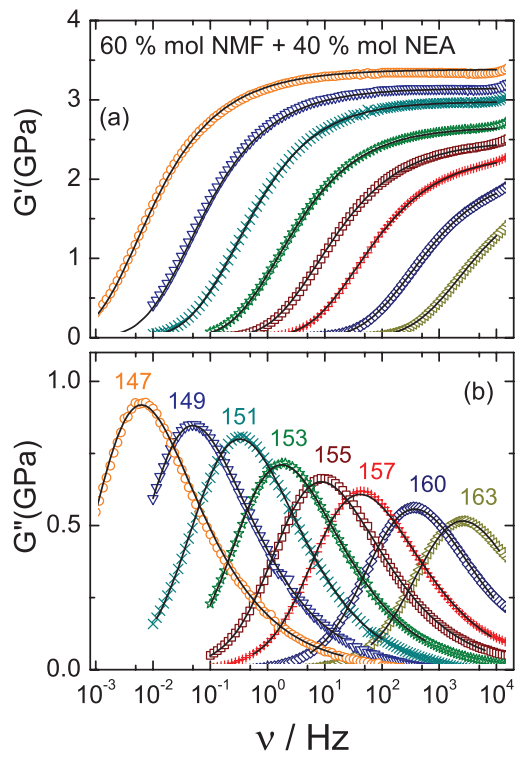

FIG. 3. (a) The real and (b) the imaginary part of the shear modulus as a function of frequency for a $60 \mathrm{~mol} . \% \mathrm{NMF}$ and 40 mol.\% NEA mixture. The numbers indicate temperatures in Kelvin and the solid lines are fits using Eqs. (9) and (10).

thors, a 60 mol.\% NMF + 40 mol.\% NEA supercooled binary system showed a reduced crystallization tendency, while the shape of its dielectric loss preserves the characteristics observed for the individual liquid constituents, comprising an enormously large Debye peak with an additional small contribution at higher frequencies.

The shear modulus for a mixture of $60 \mathrm{~mol} . \% \mathrm{NMF}$ and 40 mol.\% NEA is shown in Fig. 3 as a function of frequency for several temperatures. The high-frequency plateau in $G^{\prime}(v)$, which again is resolved only in the deeply supercooled regime, has a value $G_{\infty}(3.3 \mathrm{GPa}$ at $147 \mathrm{~K})$ that is larger than the one observed for PC. Such a relatively high value of $G_{\infty}$ is not uncommon for associating liquids. ${ }^{55}$ As observed in Fig. 3, both real and imaginary parts of the frequency-dependent shear modulus were well interpolated by Eqs. (9) and (10). For the present amide mixture timetemperature superposition does not apply: In Fig. 4(b) one sees that the slope of the scaled data changes at the highest reduced frequencies if temperature is varied. The fitting parameter $\alpha$ increases from 0.32 to 0.53 when decreasing the temperature from $163 \mathrm{~K}$ to $147 \mathrm{~K}$ and $\beta$ decreases from 0.77 to 0.47 in the same $T$ range. The mean time constants $\tau_{\text {shear }}$ for the NEA-NMF system are included in Fig. 5.

\section{DISCUSSION}

\section{A. Time constants and hydrodynamic radii}

The relaxation times for PC and for the amide mixture are presented in Fig. 5 as a function of inverse temperature. For comparison this figure includes the time constants for 2-ethyl-1-hexanol (2E1H), as taken from Ref. 43. 2E1H is a well investigated monoalcohol. ${ }^{38,41,43,56,57}$ With the NEA-NMF mixture it shares the peculiarity of exhibiting a 


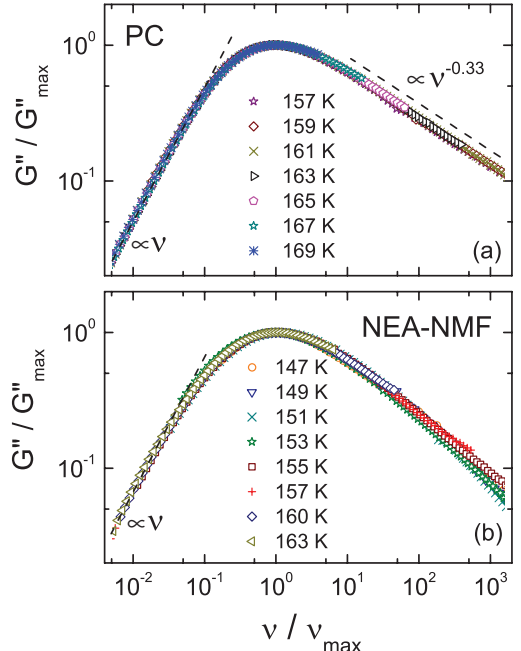

FIG. 4. Normalized shear loss spectra plotted vs reduced frequency for the glass formers (a) PC and (b) NEA-NMF. The dashed line on the left side of the peak indicates a linear frequency dependence and the one on the highfrequency flank of the peak in (a) is proportional to $v^{-0.33}$. A good scaling is achieved for the PC data indicating that time-temperature superposition applies. This is not in this case for NEA-NMF for which the high-frequency slope in $G^{\prime \prime}(\omega)$ changes with the temperature.

large monodispersive (Debye-like) dielectric feature. Finally, for PC the dielectric loss spectra were fitted with the ColeDavidson function $\varepsilon^{\prime \prime} \propto \operatorname{Im}\left[\frac{1}{\left(1+i \omega \tau_{C D}\right)^{\beta C D}}\right]$, briefly mentioned below Eq. (10), for which the dielectric time constants can be evaluated as $\tau_{\text {diel }}=\tau_{\mathrm{CD}} \beta_{\mathrm{CD}} \cdot{ }^{58}$

In harmony with previous studies on molecular glassforming liquids, at a given temperature the shear time constant is smaller than the dielectric one ${ }^{59,60}$ The ratio of the two time scales $\sigma \equiv \tau_{\text {diel }} / \tau_{\text {shear }}$ [9 for PC, 80 for NMF-NEA, and 18200 (!) for $2 \mathrm{E} 1 \mathrm{H}]$ does not change much with temperature in the commonly investigated $T$ range.

The $\sigma$ values can be used to determine the effective molecular radii (also called the hydrodynamic radii) of the relaxing constituents. ${ }^{14,61,62}$ The experimental results can be

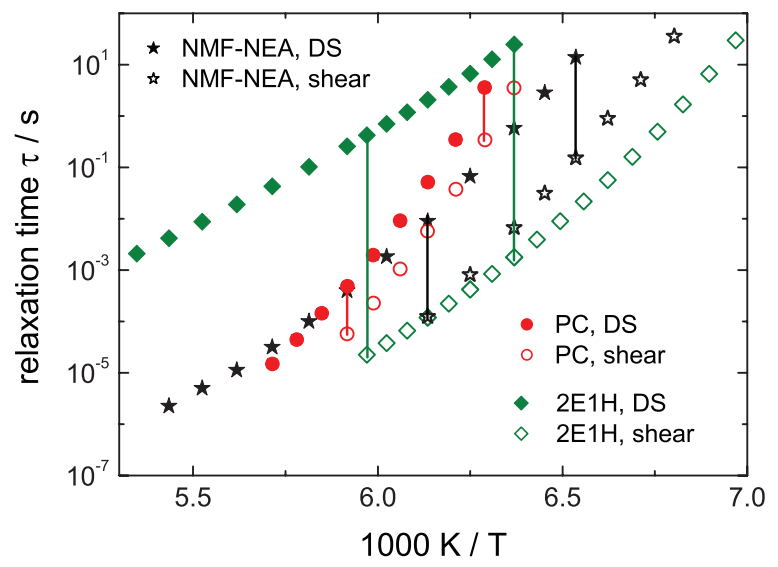

FIG. 5. Rheological and dielectric time constants for PC, NEA-NMF, and $2 \mathrm{E} 1 \mathrm{H}$ (the latter from Ref. 43) as a function of inverse temperature. For every system the two solid lines connecting the corresponding data sets are drawn to have the same length, indicating that no severe decoupling occurs between the time scales probed by the two techniques in the commonly investigated temperature range. compared with the molecular radii (also called the van der Waals radii) $R_{\mathrm{vdW}}$ estimated from atomic increments. ${ }^{63}$ The Debye-Stokes-Einstein equation $\tau_{\text {diel }}=\eta\left(4 \pi R_{H}^{3} / k_{B} T\right)$ combined with the Maxwell relation $\eta=G_{\infty} \tau_{\text {shear }}$ provide a hydrodynamic radius of $1.3 \AA$ for $\mathrm{PC}$ at $T=155 \mathrm{~K}$ and $2 \AA$ for the NMF-NEA mixture at $147 \mathrm{~K}$. Using Ref. 63 one can estimate that $R_{\mathrm{vdW}}$ is close to $3 \AA$ for PC, for NMF, as well as for NEA. Thus, for PC and for the NMF-MEA system, the experimentally determined hydrodynamic radii appear to be smaller than the calculated one, if one assumes that for the NMF-NEA mixture $R_{\mathrm{vdW}}$ is the average of the radii of the two components.

While for simple molecular liquids it is also often observed that the experimentally estimated $R_{H}$ is smaller than $R_{\mathrm{vdW}}{ }^{64}$ the situation is different for $2 \mathrm{E} 1 \mathrm{H}$. In this case, using $\sigma=18000$ and $G_{\infty}=10^{9} \mathrm{~Pa}$ (Ref. 43) one estimates $R_{H} \approx 10 \AA$ at $143.5 \mathrm{~K}$, which is much larger than $R_{\mathrm{vdW}}$ for $2 \mathrm{E} 1 \mathrm{H}$. A similar result was obtained for $2 \mathrm{E} 1 \mathrm{H}$ when comparing its dielectric time constants with self-diffusion coefficients probed by NMR. ${ }^{65}$ We remind the reader that for the evaluation of $\tau_{\text {diel }}$ and, implicitly, of $R_{H}$ we used the time constants of the main dielectric peaks, i.e., of the Debye peak in the case of NEA-NMF and of 2E1H, and of the structural relaxation peak for PC.

\section{B. Conversion between dielectric permittivity and shear modulus}

In the following, we test the conversion formulae given by Eqs. (6) and (7) for PC, TPG, and NEA-NMF. The parameters $\Delta \varepsilon, \varepsilon_{\infty}, G_{\infty}$, and the optimized $\lambda$ used for the transformations are given in Table I, where the values calculated for $A, B$, and $\lambda_{\min }$ are also included.

In Figs. 6-8 we show dielectric loss spectra together with the converted data using the real and the imaginary part of the complex shear modulus recorded in a common temperature range for all three substances. One recognizes that, for the $\lambda$ values given in Table I [in all cases below $\lambda_{\min }$, cf. Eq. (8)], not only the peak positions but also the spectral shapes are nicely reproduced.

For the calculation of $\varepsilon^{\prime \prime}(v)$ only the parameter $B(\lambda)$ enters in Eq. (6). However, the calculation of $\varepsilon^{\prime}(v)$ also involves the parameter $A(\lambda)$. Keeping $\lambda$ as a free parameter, best coincidence of measured and converted data yields a negative $A$. As discussed in Sec. II, the condition $A \geq 0$ is fulfilled for $\lambda \geq \lambda_{\text {min }}$.

As observed for PC in Fig. 6(b) the dielectric loss curve re-calculated at $161 \mathrm{~K}$ for $\lambda=\lambda_{\min }$ departs from the measured $\varepsilon^{\prime \prime}(v)$, mainly regarding the time scale. However, such quantitative inadequacies are not surprising in view of the simplicity of our model. The sensitivity of the transformation on $\lambda$ can also be inferred from Fig. 6(c). Here, Eq. (7) was employed for converting the dielectric data at $161 \mathrm{~K}$ to the imaginary part of the shear modulus. Using $\lambda=\lambda_{\min }$, one obtains a $G^{\prime \prime}(v)$ curve which is shifted with respect to the measured shear loss. With the parameter $A$ set to $n^{2} \approx 2$ ( $n$ being the refraction index at room temperature), i.e., using the condition that was imposed on the transformation in Ref. 28, not even a peak in the calculated $G^{\prime \prime}(v)$ curve is obtained (not shown). 
TABLE I. The parameters used for converting the data from shear modulus to dielectric permittivity. For all three substances the optimal $\lambda$ is below $\lambda_{\text {min }}$, corresponding to a capacitance $C_{1}<0$.

\begin{tabular}{lccccccc}
\hline \hline Glass former & $T(\mathrm{~K})$ & $\varepsilon_{\infty}$ & $\Delta \varepsilon$ & $G_{\infty}(\mathrm{GPa})$ & $A$ & $B$ & $\lambda_{\min }\left(\mathrm{Pa}^{-1}\right)$ \\
\hline PC & 171 & 3.5 & 93 & 1.21 & -12.19 & 108.6 & $2.3 \times 10^{-10}$ \\
& 169 & & 94.6 & 1.24 & -11.96 & 110 & $2.23 \times 10^{-10}$ \\
& 167 & & 95.6 & 1.26 & -11.67 & 110.8 & $2.18 \times 10^{-10}$ \\
& 165 & & 96.6 & 1.28 & -11.46 & 111.6 & $2.14 \times 10^{-10}$ \\
& 163 & & 97.7 & 1.33 & -11.05 & 112.2 & $2.08 \times 10^{-10}$ \\
& 161 & & 98.8 & 1.39 & -10.52 & 112.8 & $1.99 \times 10^{-10}$ \\
& 159 & & 98.8 & 1.46 & -9.93 & 112.2 & $1.89 \times 10^{-10}$ \\
& 157 & & 99 & 1.5 & -9.61 & 112.1 & $1.84 \times 10^{-10}$ \\
& 155 & & 100 & 1.6 & -8.86 & 112.4 & $1.73 \times 10^{-10}$ \\
TPG & 200 & 2.7 & 20 & 2.5 & -0.21 & 22.9 & $1.31 \times 10^{-10}$ \\
& 192 & & 22 & 2.6 & -0.24 & 24.9 & $1.32 \times 10^{-10}$ \\
NEA-NMF & 163 & 3.5 & 424 & 2 & -3.53 & 431 & $1.41 \times 10^{-10}$ \\
& 160 & & 435 & 2.22 & -2.85 & 441 & $1.28 \times 10^{-10}$ \\
& 157 & & 445 & 2.3 & -2.63 & 451 & $1.23 \times 10^{-10}$ \\
& 155 & & 450 & 2.55 & -2.03 & 455.5 & $1.11 \times 10^{-10}$ \\
& 153 & & 455 & 2.67 & -1.79 & 460.3 & $1.06 \times 10^{-10}$ \\
\hline \hline
\end{tabular}

On the other hand, the agreement improves considerably if the conversion procedure is not restricted to $A \geq 0$ or equivalently to $\lambda \geq \lambda_{\min }$. The authors of Refs. 15 and 28 also noted that acceptable fits can be obtained if all (including negative) values of the index of refraction are allowed for. A negative capacitance may sound odd, but as an abstract circuit element it is not forbidden and it is not necessarily unphysical, as has been discussed in various instances ${ }^{66,67}$ For example, in some circuit models of ferroelectric behavior the formal introduction of a negative capacitance was used to reflect the fact that the free energy as a function of the polarization $P$ develops a

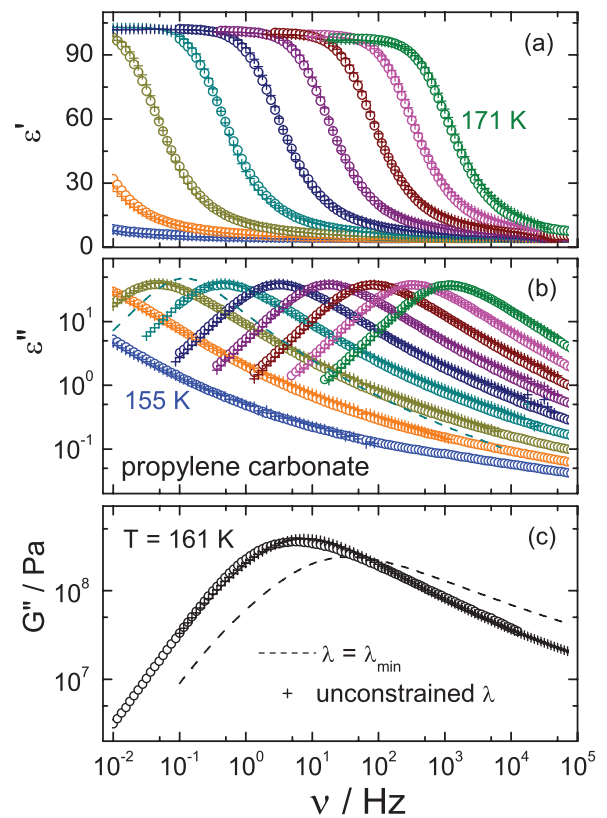

FIG. 6. The shear data for PC, measured every $2 \mathrm{~K}$ between $171 \mathrm{~K}$ and $155 \mathrm{~K}$, is converted via Eq. (6) (crosses) and compared to the measured (a) real and (b) imaginary part of the complex permittivity (open circles). In frame (b) the dashed line represents the calculation of the dielectric loss at $T=161 \mathrm{~K}$ for $\lambda=\lambda_{\min }$. (c) The inverse transformation, via Eq. (7), is applied to the dielectric data of PC for $T=161 \mathrm{~K}$. The dashed line is calculated for $\lambda=\lambda_{\min }$. negative curvature at $P=0$ (see Ref. 67). In the present situation the origin of the negative value of $C_{1}$ remains unclear. Given the surprisingly good quantitative fits of the converted and the measured data, it seems that the idea of some element of parallel dipolar alignment being present in supercooled liquids with large dielectric strength (usually cast into the form of a Kirkwood factor $g>1$, see Ref. 1) may be worth pursuing in future.

Consistent with previous studies, our results demonstrate that the excess wing can be detected not only via dielectric, light scattering, and NMR measurements ${ }^{8}$ but also in mechanical shear spectra. While an excess wing is directly discernible in the shear loss data of PC [see Fig. 2(b)], its observation for the other systems is probably hampered by the broader loss

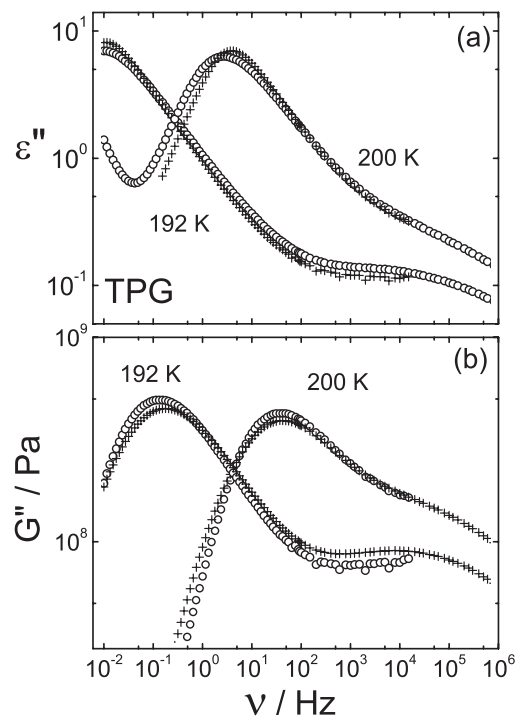

FIG. 7. (a) The shear data of tripropylene glycol (TPG), measured at two temperatures, ${ }^{59}$ are transformed via Eq. (6) (crosses) and compared with the imaginary part of the dielectric permittivity (open circles). In frame (b) the inverse transformation, accomplished via Eq. (7), is applied to the dielectric data plotted in (a) and the results (crosses) are compared with the measured shear loss (open circles). 


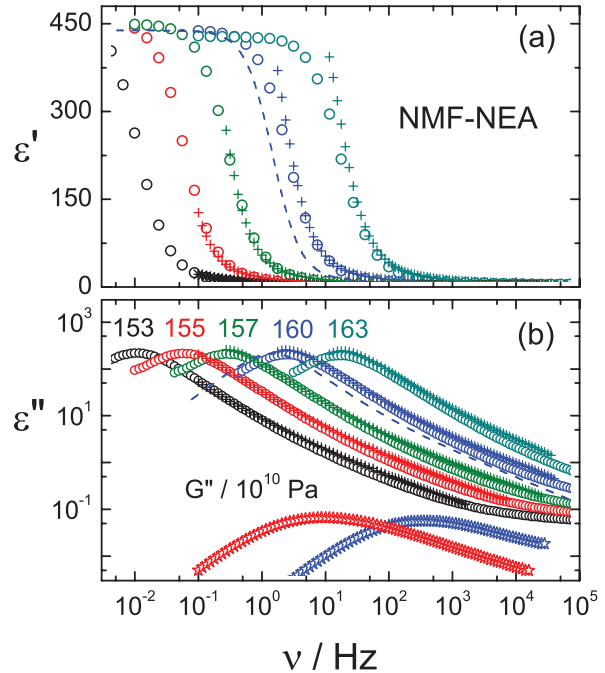

FIG. 8. The transformations (crosses) via Eq. (6) are compared with (a) the real and (b) the imaginary parts of the dielectric permittivity (open circles) for the glass-forming system NEA-NMF. The dashed lines are calculations for $\lambda=\lambda_{\min }$ at $T=160 \mathrm{~K}$. In (b) the loss shear data, divided by a factor $10^{10}$, are shown for the two temperatures $155 \mathrm{~K}$ and $160 \mathrm{~K}$.

peak of the shear response as compared to its dielectric counterpart. The fact that the conversion works well in the entire frequency range including dynamics faster than the $\alpha$-process indicates that secondary processes like the excess wing are not to be regarded as merely dielectric features.

In order to test the current approach for a different scenario, the transformations expressed by Eqs. (6) and (7) were also applied to the dielectric and shear data reported in Ref. 59 for TPG. Like PC and NMF-NEA, this system exhibits a fairly large dielectric strength $(\Delta \varepsilon=20$ at $T=200 \mathrm{~K})$ but, different from these two other liquids, its dielectric response clearly indicates the presence of a secondary relaxation peak. As Fig. 7 illustrates, the unconstrained conversion of the data from shear to dielectric and from dielectric to shear - works again very well for the entire spectra including the $\beta$-peak.

The fact that a single electro-viscoelastic parameter $\lambda$ controls the shapes of both the main and the secondary process (excess wing and/or $\beta$-process) is in harmony with the view that essentially the same relaxing unit, probing the same local environment, is involved in both processes. ${ }^{33,68,69}$

Figures 8(a) and 8(b) show the conversion for the NEANMF system. The shear modulus data, measured in a more limited frequency range than the dielectric ones, were transformed via Eq. (6) to dielectric spectra that are dominated by a Debye-like peak. The calculated dielectric spectra reveal an overall shape that is quite similar to the measured one, for both constrained and unconstrained $\lambda$. As demonstrated next, for this amide mixture the presence of a "single relaxation time" peak in the calculated spectrum is due to the large value of its dielectric strength.

In Fig. 9 we illustrate the effect of varying the dispersion strength $\Delta \varepsilon$ on the dielectric loss as modeled on the basis of Eq. (5). According to this figure, a fairly large value of $\Delta \varepsilon$ induces a peak in the dielectric spectrum that (i) is mono-exponential; (ii) is slower than the corresponding shear peak; and (iii) becomes gradually larger and separates more and more from the structural process when $\Delta \varepsilon$ is increased.

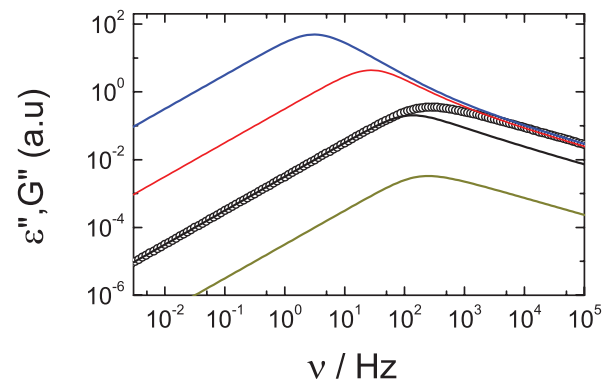

FIG. 9. A calculated shear loss spectrum (open circles) with a highfrequency exponent of 0.5 is used as input for the calculations of $\varepsilon^{\prime \prime}$ on the basis of Eq. (6) with $\lambda=1, \varepsilon_{\infty}=3$, and $\Delta \varepsilon=0.1$ (lowest line). For the other lines we used $\Delta \varepsilon=1, \Delta \varepsilon=10$, or $\Delta \varepsilon=100$ (upper lines).

As a consequence of item (iii), for systems with large $\Delta \varepsilon$ the $\alpha$-relaxation can be masked, i.e., it merely appears as a high-frequency shoulder on the dominant peak, as is observed in the loss spectra of secondary amides and neat monoalcohols. Thus, the appearance of a separate, slow Debye peak in the calculated dielectric response of NEA-NMF might be justified by the circuit model of Fig. 1 in conjunction with a large value for $\Delta \varepsilon$ (above 400), whatever the reason for such a dispersion strength is. Large $\Delta \varepsilon$ 's can be due, e.g., to the formation of chain-like hydrogen bonded structures featuring a large end-to-end dipole moment. ${ }^{40,70,71}$ With such a dipole moment fluctuating on a time scale longer than the one characteristic for the structural relaxation, the situation resembles the one known from type A polymers. ${ }^{72}$ All in all, the simple circuit model captures several aspects of the complex relaxation pattern for systems with large dielectric relaxation strength.

\section{Tests of the shoving model}

To further assess possible common features or differences between NEA-NMF and PC, we next discuss the temperature dependence of their relaxation times as predicted by the shoving model. ${ }^{27}$ In this model the viscosity (or the shear relaxation time) of a supercooled liquid is given by its temperature dependent instantaneous shear modulus $G_{\infty}(T)$ via the expression

$$
\tau=\tau_{0} \exp \left[\frac{V_{c} G_{\infty}(T)}{k_{B} T}\right]
$$

In this model the product $V_{c} G_{\infty}$ represents the free energy barrier, which is calculated as the reversible elastic work done in shoving aside the surrounding molecules in order to reduce the local density. The microscopic volume $V_{c}$ affected by a flow event is assumed to be temperature independent. In Eq. (11) the pre-exponential factor is given by the relation $\tau_{0}=1 /\left(2 \pi v_{0}\right)$ with $v_{0}$ denoting a vibrational frequency. The shoving model was successfully applied to describe the nonArrhenius temperature dependence of the relaxation times of several neat supercooled organic liquids, ${ }^{59,60}$ but so far was not tested for binary mixtures.

From Eq. (11) one notes that $\tau \rightarrow \tau_{0}$ if $G_{\infty} / T \rightarrow 0$ (Fig. 10). If time-temperature superposition applies for the 


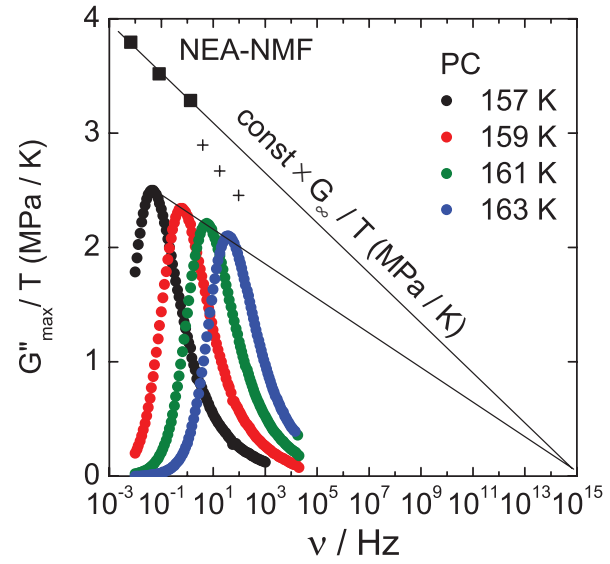

FIG. 10. This figure shows that $G_{\infty} / T$ for PC and NEA-NMF measured at $147 \mathrm{~K}, 149 \mathrm{~K}$, and $151 \mathrm{~K}$ extrapolates to zero at a frequency close to $10^{15} \mathrm{~Hz}$, in agreement with the predictions of the shoving model. For NEANMF, at temperatures above $151 \mathrm{~K}$, the data show deviations from the behavior predicted by Eq. (11), presumably due to the onset of crystallization.

shear loss spectra, i.e., if their shapes are temperature independent, $G_{\infty}$ is proportional to the maximum value of $G^{\prime \prime}(v)$. Thus, according to the shoving model, the maximum in $G^{\prime \prime}(v) / T$ approaches zero at frequencies close to $v_{0} .{ }^{27}$ This prediction is tested for PC in Fig. 10. For this liquid the line that connects the maxima of the $G^{\prime \prime}(v) / T$ curves indeed extrapolates to zero at typical vibrational frequencies of about $10^{15}$ $\mathrm{Hz}$. This model-independent procedure cannot be applied for the NEA-NMF mixture because time-temperature superposition does not apply here, see Sec. IV. To circumvent this problem in Fig. 10 we included the values for $G_{\infty} / T$ for this liquid with $G_{\infty}$ taken from the fitting procedure described in Sec. IV. These data are divided by a factor of 6 for the sake of representation clarity. At the lowest three temperatures, $G_{\infty} / T$ extrapolates to zero again close to a vibrational frequency, demonstrating the applicability of the shoving model for this hydrogen-bonded mixture in this $T$-range. At higher temperature deviations occur from the expected behavior. Since the shear data were recorded upon increasing the temperature, the monotonic decrease of the area below the loss spectra could be attributed to a progressive crystallization of this sample upon heating.

\section{CONCLUDING REMARKS}

We performed an experimental investigation of a simple glass former (propylene carbonate) and an associating liquid (a mixture of two secondary amides) with shear and with dielectric spectroscopy. The time constants obtained via these techniques provide effective molecular radii for the two systems that are somewhat smaller than the theoretically estimated ones. The similarities between the broadband rheological and the dielectric spectral shapes for these two systems and for the previously experimentally investigated tripropylene glycol were discussed using a simple electrical circuit approach that allows one to convert the mechanical into dielectrical data and vice versa. The model involves a circuit formed by two capacitors and one frequency dependent impedance proportional to the dynamic viscosity of the inves- tigated material, a model that is mathematically equivalent to the GDB model. The proportionality constant $\lambda$, which defines the strength of the electro-viscoelastic coupling, is the only adjustable parameter within that approach if the experimentally determined high- and low-frequency dielectric constants are imposed.

Allowing for a negative value of the capacitance $C_{1}$ we achieved excellent agreement between the model calculations and the experimental data for the entire dynamics, including the $\alpha$-relaxation and the secondary processes for PC and TPG, as well as the strong Debye-like process for the secondary amides. We suggested that a negative capacitance may not be unphysical, but that it might reflect the tendency for a preferred molecular alignment. Also for materials with high dielectric relaxation strength, such as the secondary amides, the circuit model gives a qualitatively correct account of several features of the dominant dielectric process. Finally, the predictions of the shoving model were tested, showing that the applicability of this model can be extended to include not just neat liquids but also binary mixtures of hydrogen-bonding compounds.

\section{ACKNOWLEDGMENTS}

We are indebted to Kristine Niss, Bo Jakobsen, and Robert Kahlau for useful discussions and constructive comments regarding the paper. Partial funding by the Deutsche Forschungsgemeinschaft via Grant No. Bo1301/8-1 is gratefully acknowledged. The centre for viscous liquid dynamics Glass and Time is sponsored by the Danish National Research Foundation (DNRF).

${ }^{1}$ C. J. F. Böttcher and P. Bordewijk, Theory of Electric Polarization I: Dielectrics in Static Fields (Elsevier, Amsterdam, 1978).

${ }^{2}$ Broadband Dielectric Spectroscopy, edited by F. Kremer and A. Schönhals (Springer, Heidelberg, 2003).

${ }^{3}$ G. P. Johari, Ann. N.Y. Acad. Sci. 279, 117 (1976).

${ }^{4}$ Disorder Effects on Relaxational Processes: Glasses, Polymers, Proteins, edited by R. Richert and A. Blumen (Springer, Berlin, 1994).

${ }^{5}$ G. Williams, J. Non-Cryst. Solids 131-133, 1 (1991); Materials Science and Technology, edited by R. W. Cahn, P. Haasen, and E. J. Kramer (Verlag Chemie, Weinheim, 1993), Vol. 12.

${ }^{6}$ J. E. Anderson, Faraday Symp. Chem. Soc. 6, 82 (1972); G. Hinze, Phys. Rev. E 57, 2010 (1998); M. S. Beevers, J. Crossley, D. C. Garington, and G. Williams, J. Chem. Soc., Faraday Trans. 2 72, 1482 (1976).

${ }^{7}$ A. Brodin, C. Gainaru, V. Porokhonskyy, and E. A. Rössler, J. Phys.: Condens. Matter 19, 205104 (2007).

${ }^{8}$ C. Gainaru, O. Lips, A. Troshagina, R. Kahlau, A. Brodin, F. Fujara, and E. A. Rössler, J. Chem. Phys. 128, 174505 (2008).

${ }^{9}$ T. Blochowicz, A. Kudlik, S. Benkhof, J. Senker, and E. Rössler, J. Chem. Phys. 110, 12011 (1999).

${ }^{10}$ H. Z. Cummins, G. Li, W. Du, R. M. Pick, and C. Dreyfus, Phys. Rev. E 53, 896 (1996).

${ }^{11}$ T. Blochowicz, A. Brodin, and E. A. Rössler, Adv. Chem. Phys. 133(Part A), 127 (2006).

${ }^{12}$ A. Brodin, E. A. Rössler, R. Bergman, and J. Mattson, Eur. Phys. J. B 36, 349 (2003).

${ }^{13}$ R. Böhmer, G. Diezemann, G. Hinze, and E. Rössler, Prog. Nucl. Magn. Reson. Spectrosc. 39, 191 (2001).

${ }^{14}$ K. Schröter and E. Donth, J. Non-Cryst. Solids 307, 270 (2002).

${ }^{15}$ T. Christensen and N. B. Olsen, J. Non-Cryst. Solids 172, 357 (1994).

${ }^{16}$ R. Zorn, F. I. Mopsik, G. B. McKenna, L. Willner, and D. Richter, J. Chem. Phys. 107, 3645 (1997).

${ }^{17}$ N. G. McCrum, B. E. Read, and G. Williams, Anelastic and Dielectric Effects in Polymeric Systems (Dover, New York, 1967). 
${ }^{18}$ J. D. Ferry, Viscoelastic Properties of Polymers, 3rd ed. (Wiley, New York, 1980).

${ }^{19}$ J. C. Dyre, Rev. Mod. Phys. 78, 953 (2006).

${ }^{20}$ A. Gemant, Trans. Faraday Soc. 31, 1582 (1935).

${ }^{21}$ E. A. DiMarzio and M. Bishop, J. Chem. Phys. 60, 3802 (1974).

${ }^{22}$ P. Debye, Polar Molecules (The Chemical Catalog Company, New York, 1929).

${ }^{23}$ R. Díaz-Calleja, E. Riande, and J. S. Román, J. Polym. Sci. Pol. Phys. 31, 711 (1993).

${ }^{24} \mathrm{~K}$. Niss and B. Jakobsen, M. Sc. thesis, University of Roskilde, Denmark, 2003, see http://milne.ruc.dk/ImfufaTekster/pdf/424.pdf.

${ }^{25}$ U. Buchenau, M. Ohl, and A. Wischnewski, J. Chem. Phys. 124, 094505 (2006).

${ }^{26}$ N. Hirai and H. Eyring, J. Appl. Phys. 29, 810 (1958).

${ }^{27}$ J. C. Dyre, N. B. Olsen, and T. Christensen, Phys. Rev. B 53, 2171 (1996); J. Non-Cryst. Solids 352, 4635 (2006).

${ }^{28}$ K. Niss, B. Jakobsen, and N. B. Olsen, J. Chem. Phys. 123, 234510 (2005).

${ }^{29}$ P. Lunkenheimer, U. Schneider, R. Brand, and A. Loidl, Contemp. Phys. 41, 15 (2000).

${ }^{30}$ C. Gainaru, A. Rivera, S. Putselyk, G. Eska, and E. A. Rössler, Phys. Rev. B 72, 174203 (2005)

${ }^{31}$ G. P. Johari and M. Goldstein, J. Chem. Phys. 53, 2372 (1972).

${ }^{32}$ A. Kudlik, S. Benkhof, T. Blochowicz, C. Tschirwitz, and E. Rössler, J. Mol. Struct. 479, 201 (1999)

${ }^{33}$ P. K. Dixon, L. Wu, S. R. Nagel, B. D. Williams, and J. P. Carini, Phys. Rev. Lett. 65, 1108 (1990).

${ }^{34}$ U. Schneider, R. Brand, P. Lunkenheimer, and A. Loidl, Phys. Rev. Lett. 84, 5560 (2000); K. L. Ngai, P. Lunkenheimer, C. Leon, U. Schneider, R. Brand, and A. Loidl, J. Chem. Phys. 115, 1405 (2001).

${ }^{35}$ C. Gainaru, R. Kahlau, E. A. Rössler, and R. Böhmer, J. Chem. Phys. 131, 184510 (2009).

${ }^{36}$ A. Brodin and E. A. Rössler J. Chem. Phys. 126, 244508 (2007).

${ }^{37}$ L.-M. Wang and R. Richert, J. Chem. Phys. 123, 054516 (2005).

${ }^{38}$ H. Huth, L.-M. Wang, C. Schick, and R. Richert, J. Chem. Phys. 126, 104503 (2007).

${ }^{39}$ C. Hansen, F. Stickel, T. Berger, R. Richert, and E. W. Fischer, J. Chem. Phys. 107, 1086 (1997).

${ }^{40}$ C. Gainaru, R. Meier, S. Schildmann, C. Lederle, W. Hiller, E. A. Rössler, and R. Böhmer, Phys. Rev. Lett. 105, 258303 (2010).

${ }^{41}$ C. Gainaru and R. Böhmer, J. Non-Cryst. Solids 356, 542 (2010).

${ }^{42}$ R. Kono, T. A. Litovitz, and G. E. McDuffie, J. Chem. Phys. 45, 1790 (1966).

${ }^{43}$ B. Jakobsen, C. Maggi, T. Christensen, and J. C. Dyre, J. Chem. Phys. 129, 184502 (2008).

${ }^{44}$ T. Christensen and N. B. Olsen, Rev. Sci. Instrum. 66, 5019 (1995).

${ }^{45}$ B. Igarashi, T. Christensen, E. H. Larsen, N. B. Olsen, I. H. Pedersen, T. Rasmussen, and J. C. Dyre, Rev. Sci. Instrum. 79, 045105 (2008).
${ }^{46}$ B. Igarashi, T. Christensen, E. H. Larsen, N. B. Olsen, I. H. Pedersen, T. Rasmussen, and J. C. Dyre, Rev. Sci. Instrum. 79, 045106 (2008).

${ }^{47}$ M. Doi and S. F. Edwards, The Theory of Polymer Dynamics (Oxford University Press, New York, 1986).

${ }^{48}$ T. B. Schrøder, N. P. Bailey, U. R. Pedersen, N. Gnan, and J. C. Dyre J. Chem. Phys. 131, 234503 (2009).

${ }^{49}$ N. Sağlanmak, A. I. Nielsen, N. B. Olsen, J. C. Dyre, and K. Niss, J. Chem. Phys. 132, 024503 (2010).

${ }^{50}$ F. Qi, K. U. Schug, A. Döß, S. Dupont, R. Böhmer, H. Sillescu, H. Kolshorn, and H. Zimmermann, J. Chem. Phys. 112, 9455 (2000).

${ }^{51}$ R. Kahlau, D. Kruk, T. Blochowicz, V. N. Novikov, and E. A. Rössler, J. Phys.: Condens. Matter 22, 365101 (2010).

${ }^{52}$ M. Abramowitz and I. A. Stegun, Handbook of Mathematical Functions (Dover, New York, 1972).

${ }^{53}$ S. Havriliak and S. Negami, J. Polymer. Sci. C 14, 99 (1966).

${ }^{54}$ The mean time constant can be derived as a function of the fit parameters $\tau_{0}, \alpha$, and $\beta$ according to $\langle\tau\rangle=\tau_{0} \Gamma\left(\frac{1+\beta}{\alpha}\right) / \Gamma\left(\frac{\beta}{\alpha}\right)$, see Ref. 51 .

${ }^{55}$ F. Scarponi, L. Comez, D. Fioretto, and L. Palmieri, Phys. Rev. B 70, 054203 (2004).

${ }^{56}$ S. S. N. Murthy and M. Tyagi, J. Chem. Phys. 117, 3837 (2002).

${ }^{57}$ C. Gainaru, S. Kastner, F. Mayr, P. Lunkenheimer, S. Schildmann, H. J. Weber, W. Hiller, A. Loidl, and R. Böhmer, Phys. Rev. Lett. 107, 118304 (2011).

${ }^{58}$ C. J. F. Böttcher and P. Bordewijk, Theory of Electric Polarization II: Dielectrics in Time-Dependent Fields (Elsevier, Amsterdam, 1978).

${ }^{59}$ B. Jakobsen, K. Niss, and N. B. Olsen, J. Chem. Phys. 123, 234511 (2005).

${ }^{60}$ C. Maggi, B. Jakobsen, T. Christensen, N. B. Olsen, and J. C. Dyre, J. Phys. Chem. B 112, 16320 (2008).

${ }^{61}$ I. Chang and H. Sillescu, J. Phys. Chem. B 101, 8794 (1997).

${ }^{62}$ N. Menon, S. R. Nagel, and D. C. Venerus, Phys. Rev. Lett. 73, 963 (1994).

${ }^{63}$ J. T. Edward, J. Chem. Educ. 47, 261 (1970).

${ }^{64}$ E. Rössler, Phys. Rev. Lett. 65, 1595 (1990).

${ }^{65}$ S. Schildmann, A. Reiser, R. Gainaru, C. Gainaru, and R. Böhmer, J. Chem. Phys. 135, 174511 (2011).

${ }^{66}$ A. K. Jonscher, J. Chem. Soc., Faraday Trans. 2 82, 75 (1986).

${ }^{67}$ S. Salahuddin and S. Datta, Nano Lett. 8, 405 (2008); A. I. Khan, D. Bhowmik, P. Yu, S. J. Kim, X. Pan, and S. Salahuddin, Appl. Phys. Lett. 99, 113501 (2011)

${ }^{68}$ R. V. Chamberlin, Phys. Rev. Lett. 82, 2520 (1999).

${ }^{69}$ R. Hilfer, Chem. Phys. 284, 399 (2002); J. Phys.: Condens. Matter 14, 2297 (2002).

${ }^{70}$ S. J. Bass, W. I. Nathan, R. M. Meighan, and R. H. Cole, J. Phys. Chem. 68, 509 (1964)

${ }^{71}$ M. Huelsekopf and R. Ludwig, Magn. Reson. Chem. 39, S127 (2001); R. Ludwig, O. Reis, R. Winter, F. Weinhold, and T. C. Farrar, J. Phys. Chem. B 102, 9312 (1998).

${ }^{72}$ G. Strobl, The Physics of Polymers (Springer, Berlin, 1997). 